\title{
Microscopic origin of the logarithmic relaxation in molecular glass-forming liquids
}

\author{
Changjiu Chen, ${ }^{1,}{ }^{*}$ Rithin P. Krishnan, ${ }^{1}$ Kaikin Wong, ${ }^{1}$ Dehong Yu, ${ }^{2}$ Fanni Juranyi, ${ }^{3}$ and Suresh M. Chathoth ${ }^{1, \dagger}$ \\ ${ }^{1}$ Department of Physics, City University of Hong Kong, Hong Kong, P. R. China \\ ${ }^{2}$ Australian Nuclear Science and Technology Organization, Lucas Heights, NSW 2234, Australia \\ ${ }^{3}$ Laboratory for Neutron Scattering and Imaging, Paul Scherrer Institute, 5232 Villigen PSI, Switzerland
}

(Received 12 June 2017; revised manuscript received 22 August 2018; published 27 September 2018)

\begin{abstract}
Logarithmic relaxation is a unique relaxation process exhibited by a few molecular liquids and biomolecules. However, the microscopic origin of logarithmic relaxation is still unclear. To understand the origin of this process, we studied two liquids that exhibit logarithmic relaxation in a dissolved state using quasielastic neutron scattering (QENS) and depolarized dynamic light scattering (DDLS). Although the intermolecular potential of the liquids is drastically different in the dissolved state from the bulk liquids, we observed that the logarithmic relaxation still persists. Our results indicate that the intermolecular potential does not play a role in determining the logarithmic relaxation process. The coupling of rotational and translational relaxation processes could be the origin of the logarithmic relaxation process exhibited by the molecular liquids.
\end{abstract}

DOI: 10.1103/PhysRevB.98.094203

\section{INTRODUCTION}

After many decades of extensive study, there is no generally accepted and fundamentally understood mechanism by which a liquid is transformed into a metastable glassy state [1]. We know that irrespective of the nature of microscopic structure or interatomic/molecular bonding a wide variety of liquids can be transformed into glassy states upon cooling below the glass-transition temperature $\left(T_{g}\right)$ [2,3]. Glassforming liquids are generally classified as strong and fragile, depending on how the viscosity changes when cooling toward $T_{g}$ [1]. A fragile liquid is characterized by non-Arrhenius behavior in the viscosity when cooling towards $T_{g}$, while the viscosity of a strong liquid displays an Arrhenius temperature dependence. The reason for the sharp increase in the viscosity of fragile liquids in a short temperature interval is still unknown. Since the viscosity is related to atomic and molecular relaxation processes, the basic features of the relaxation process have been studied intensively using a wide range of experimental techniques and molecular dynamics simulations. The main focuses of experimental studies include the frequency-dependent dielectric responses [4], timecorrelation function derived from the scattering experiments [5,6], and vibrational properties of glass-foming materials [7]. Molecular dynamics simulations of glass-formimg systems generally target the direct observation of particle motions in supercooled liquids $[8,9]$.

Unlike other experimental techniques, the self-intermediate scattering function, $\Phi(Q, t)$, measured by neutron scattering techniques can provide much more information about the relaxation processes in liquids. For glass-forming

\footnotetext{
*Present Addresses: School of Materials Science and Engineering, China University of Mining and Technology, Xuzhou 221116, P. R. China.

${ }^{\dagger}$ Corresponding Author: smavilac@ cityu.edu.hk
}

liquids, $\Phi(Q, t)$ exhibits a two-step relaxation process $[3,10]$. The first step is called the fast $\beta$-relaxation, in which atoms/molecules rattle in the cage formed by their neighboring atoms/molecules [11]. The second step is a long-range atomic/molecular diffusion process known as $\alpha$-relaxation. The fast $\beta$-relaxation process observed through $\Phi(Q, t)$ decays exponentially in most atomic/molecular liquids. Such $\Phi(Q, t)$ behavior can be well explained by the cage rattling process. However, in certain molecular liquids, the fast $\beta$ relaxation decays logarithmically with time. Examples are the orientational correlation function of 2-biphenylmethanol (BPM), phenyl-salicylate (Salol), ortho-terphenyl (OTP), and benzophenone (BZP) in their supercooled state measured by optical-Kerr-effect (OKE) experiments [12-14], and the $\Phi(Q, t)$ of RNA and proteins observed by quasielastic neutron scattering (QENS) [15,16].

The microscopic origin of the logarithmic relaxation is still unclear, and molecular liquids that exhibit this process are highly fragile. One of the striking features of these liquids is their complex molecular structure. For example, the molecular structures BPM, Salol, OTP, and BZP are quite similar and consist of two benzene rings at either end of a linear chain. This indicates that the exotic logarithmic relaxation process might be structural in origin, i.e., due to a dumbbell like structure. The OKE and light-scattering spectra of Salol, BZP, and OTP show the logarithmic relaxation process on a time scale of 20-200 ns. A schematic model of mode-coupling theory (MCT), which describes the dynamics by two correlators, one referring to density fluctuations and the other to the reorientational fluctuations of the molecules, resulted in strong rotation-translation coupling reproduced logarithmic decay of correlation, which was in excellent agreement with measured data $[11-13,16-18]$. The logarithmic decay is then explained as a manifestation of the $\beta$-peak phenomenon exhibited by molecules with large elongation $[17,19]$. The logarithmic fast $\beta$-relaxation process is also considered to originate from a unique intermolecular potential. Logarithmic relaxation is ob- 

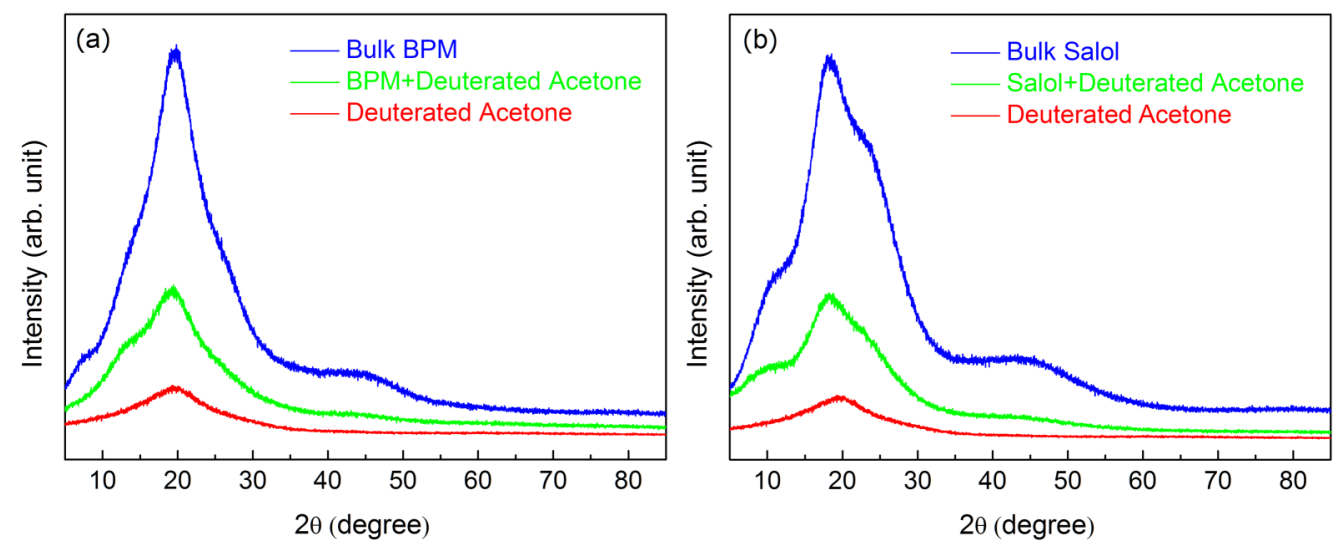

FIG. 1. (left) The wide angle diffraction patterns of bulk BPM, BPM+Deuterated acetone mixture and bulk Deuterated acetone. (right) The wide angle diffraction patterns of bulk Salol, Salol+Deuterated acetone mixture and bulk Deuterated acetone.

served in hard-sphere colloidal systems with molecular interaction potential that can be represented by hard-core repulsion and short-range attraction [14,20]. To understand the origin of the logarithmic relaxation process exhibited by molecular liquids, we have explored the relaxation processes of Salol and BPM in deuterated acetone. By dissolving the BPM or Salol in deuterated acetone the intermolecular potential in the two samples is significantly modified as that of the bulk Salol or BPM. We used QENS and depolarized dynamic light scattering (DDLS) techniques to explore the relaxation dynamics of Salol and BPM in their bulk states as well as in the dissolved state in deuterated acetone.

\section{EXPERIMENTS}

\section{A. Sample preparation}

BPM (2-biphenylmethanol, $\mathrm{C}_{13} \mathrm{H}_{12} \mathrm{O}, T_{m}=320 \mathrm{~K}, T_{g} \sim$ $239 \mathrm{~K}$ ) [21] and Salol (phenyl salicylate, $\mathrm{C}_{13} \mathrm{H}_{10} \mathrm{O}_{3}, T_{m}=$ $315 \mathrm{~K}, T_{g} \sim 218 \mathrm{~K}$ ) [22] were obtained from Sigma-Aldrich in crystalline form. For dissolved BPM and Salol samples, we made solutions of BPM or Salol with deuterated acetone using a molar ratio of 1:4 (BPM or Salol: Deuterated acetone). The BPM and Salol were soluble in deuterated acetone as seen from the X-ray diffraction pattern (Fig. 1). The deuterated acetone was chosen due to the relatively small value of the incoherent neutron cross-section of deuterium (2.05 barn) compared with that of hydrogen (80.26 barn).

\section{B. Quasielastic neutron scattering measurements}

The quasielastic neutron scattering (QENS) experiments for the dissolved BPM and Salol were performed on the PELICAN time-of-flight spectrometer at the Australian Nuclear Science and Technology Organization [23]. The neutron wavelength was $6.0 \AA$ with a resolution of $\sim 75 \mu \mathrm{eV}$. The accessible $Q$ range was $0.6-1.6 \AA^{-1}$, and the experimental setup covered a dynamical time window of $0.1-15$ ps. The QENS data were collected at five different temperatures from $T=213$ to $273 \mathrm{~K}$ in $15 \mathrm{~K}$ intervals. In addition, the samples were measured at $100 \mathrm{~K}$ to obtain the instrumental resolution function. In time-of-flight neutron scattering experiments, although the samples contain elements with coherent scattering cross section, the intensity to the scattered signals at low $Q$ values are dominated by the incoherent scattering contribution [24,25]. In the dissolved BPM and Salol samples, the incoherent neutron scattering signals of $\mathrm{C}(0.001 \mathrm{barn})$ and $\mathrm{O}(0.0008$ barn $)$ are negligible. As mentioned the incoherent neutron cross section of hydrogen is much larger than that of deuterium. Neutron scattering signals in our experiments are predominately due to incoherent scattering from the hydrogen atoms in the dissolved BPM solution $(95.14 \%)$ and dissolved Salol solution (94.22\%). In all the experiments, the data were collected for $3 \mathrm{~h}$ at each temperature. Empty sample containers were also measured at each temperature, and scattering from a vanadium sample with similar sample geometry was used to normalize the scattered signals for detector efficiency correction. The raw data were corrected for detector efficiency, self-absorption, and container scattering to obtain dynamic structure factors. The large array manipulation program (LAMP) [26] software packages were used for data reduction.

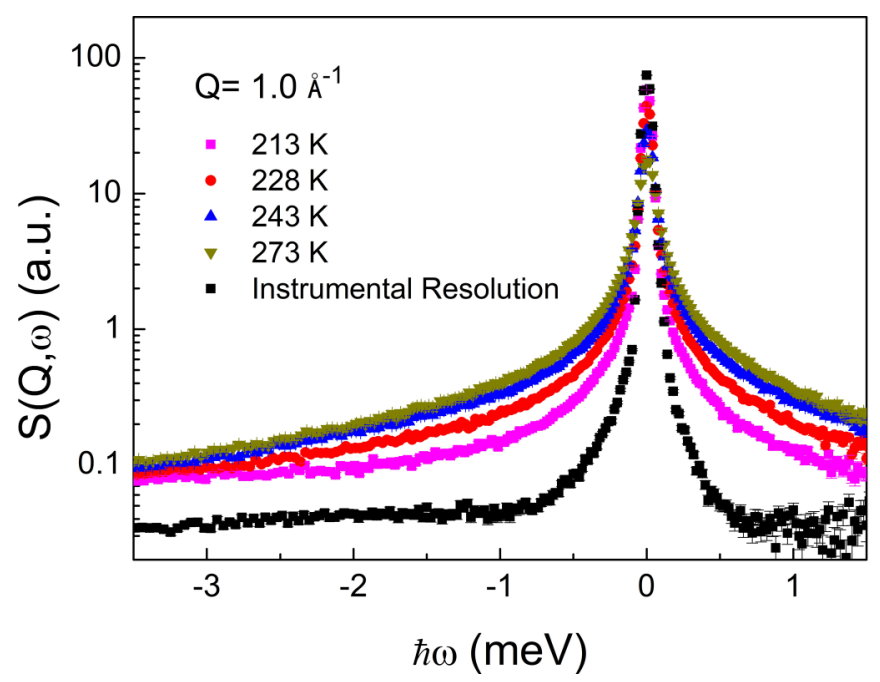

FIG. 2. The dynamic structure factors of dissolved BPM in deuterated acetone obtained on PELICAN, time-of-flight neutron spectrometer at different temperatures. 

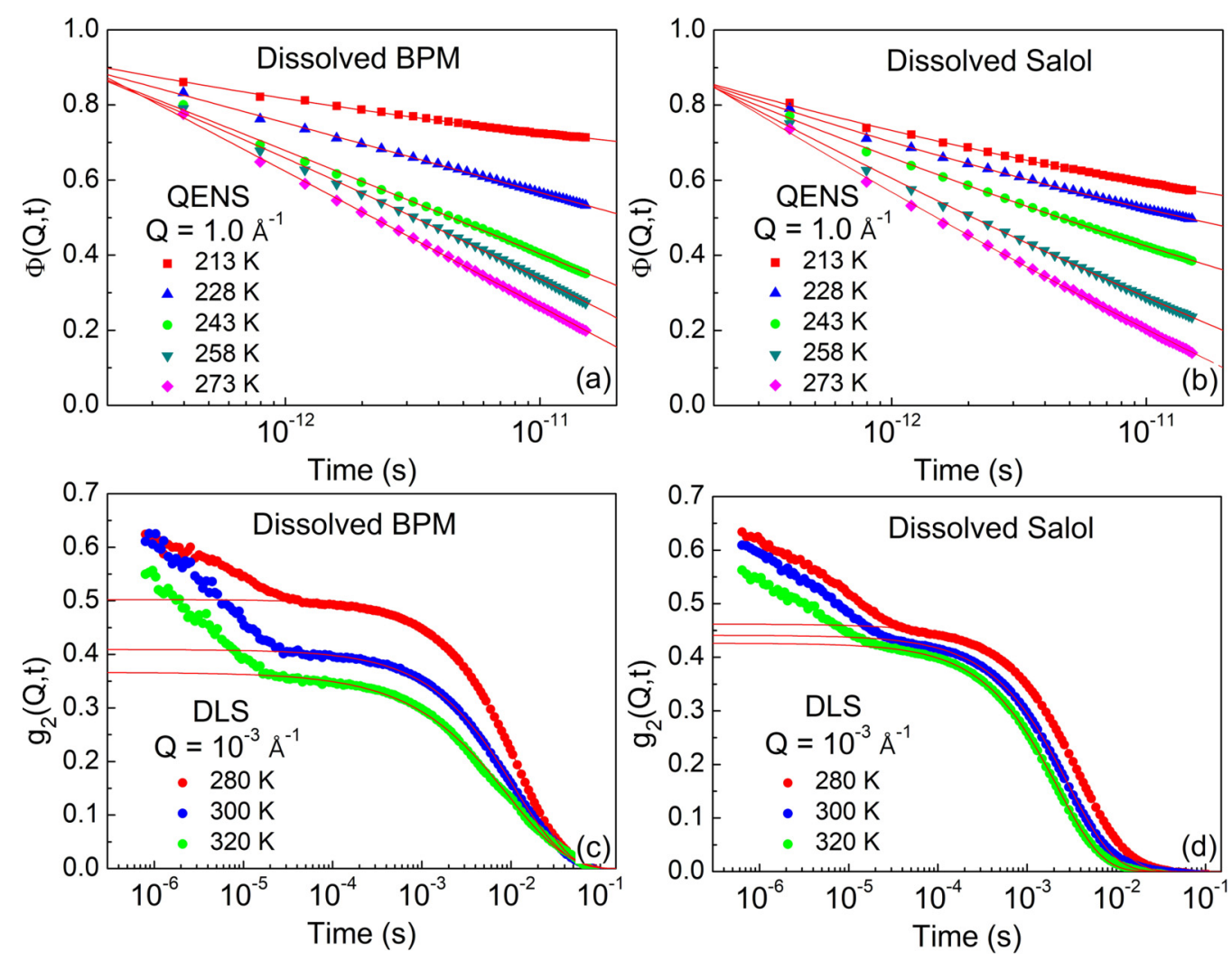

FIG. 3. (a),(b) The self-intermediate scattering functions of BPM and Salol in deuterated acetone obtained by QENS on PELICAN, timeof-flight neutron scattering spectrometer, respectively. (c),(d) The intensity-intensity autocorrelation functions obtained by DLS for dissolved BPM and Salol, respectively. The solid lines are the fits with Eqs. (1) and (3) on the self-intermediate scattering functions and intensity-intensity autocorrelation functions, respectively.

\section{High-resolution quasielastic neutron scattering measurements}

The high-resolution QENS experiments for bulk BPM were performed on the MARS high-resolution indirect timeof-flight backscattering spectrometer at SINQ, Switzerland [27]. The neutron wavelength available on this instrument was $6.61 \AA$ with a resolution of $\sim 13 \mu \mathrm{eV}$. The accessible $Q$ range with this neutron wavelength and detector coverage was $0.4 \sim$ $1.7 \AA^{-1}$, and the experimental setup covered a dynamical time window of $5 \sim 150 \mathrm{ps}$. The QENS measurements were carried out at five different temperatures from 303 to $343 \mathrm{~K}$ in $10 \mathrm{~K}$ intervals. The bulk BPM was also measured at $10 \mathrm{~K}$ to obtain the instrumental resolution function. The data were collected over a duration of $6 \mathrm{~h}$ at each temperature. The data analysis and visualization environment (DAVE) [28] software was used for data reduction. For the analysis of reduced data of PELICAN and MARS, we used a software called FRIDA developed at FRM II, Munich Germany.
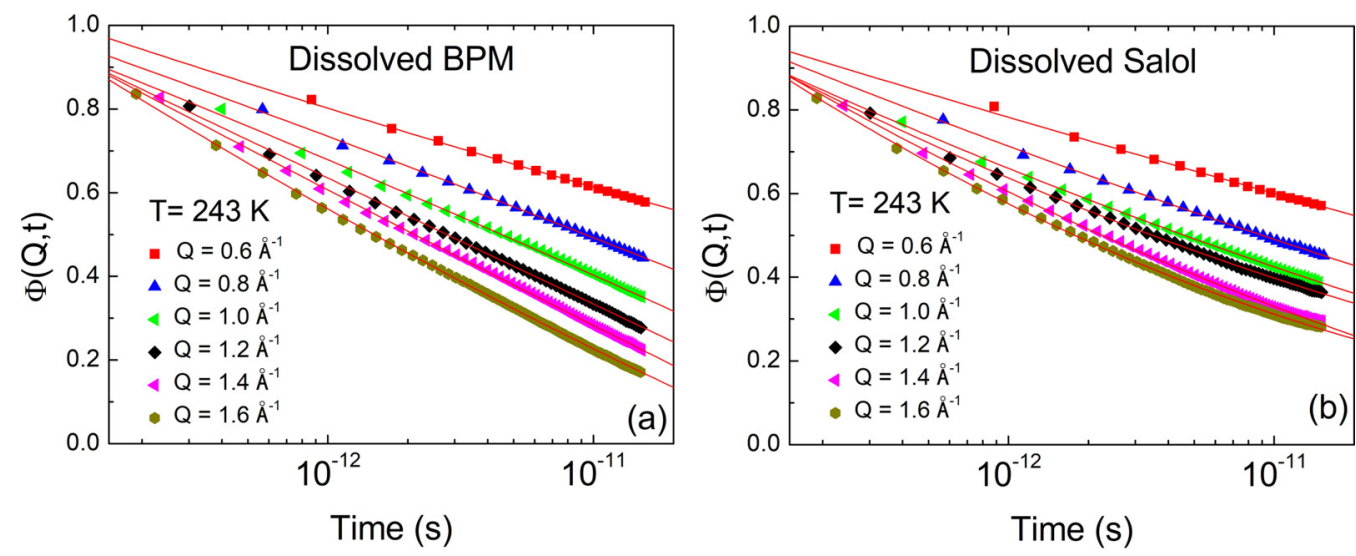

FIG. 4. The self-intermediate scattering functions of (a) dissolved BPM and (b) dissolved Salol at 243 K obtained on the PELICAN, time-of-flight neutron scattering spectrometer. 

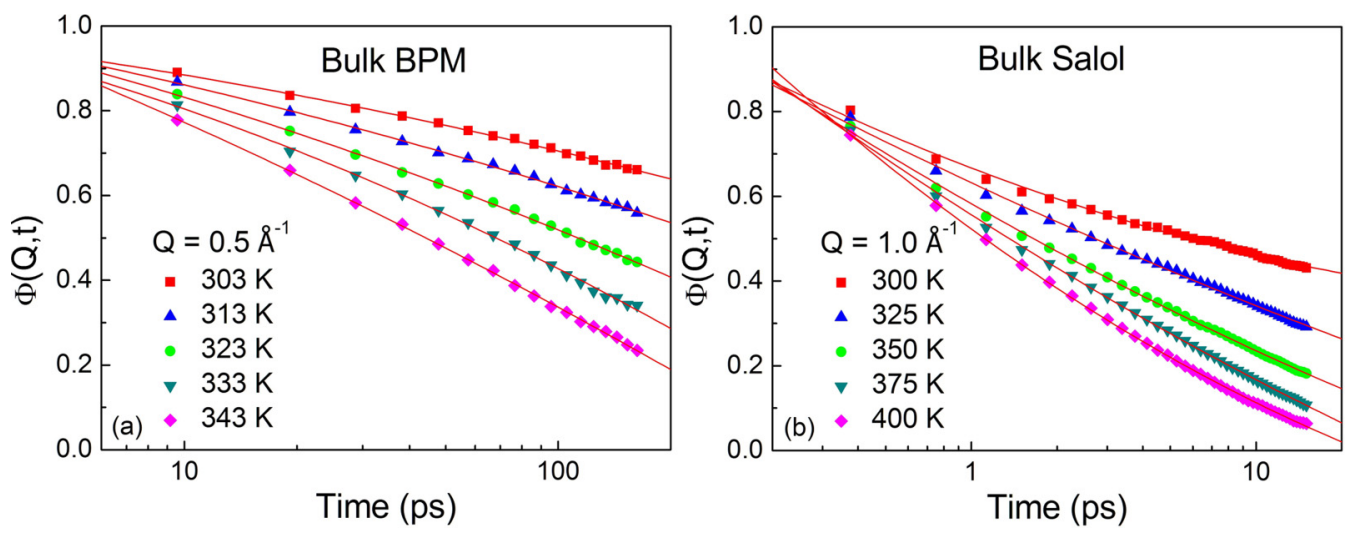

FIG. 5. (a) The self-intermediate scattering function of bulk BPM measured at different temperatures on MARS, high-resolution indirect time-of-flight neutron backscattering spectrometer. (b) The self-intermediate scattering function of bulk Salol measured at different temperatures on PELICAN, time-of-flight neutron spectrometer.

\section{Dynamic light scattering and depolarized dynamic light scattering measurements}

We used a combination of dynamic light scattering (DLS) and DDLS to study the translational and rotational diffusion coefficients of bulk BPM and Salol molecules and those molecules in a dissolved state (BPM or Salol dissolved in normal acetone with a molecular ratio of 1:4). The intensityintensity autocorrelation functions (ACFs) of DLS and DDLS were obtained by a laboratory DLS device (Photocor Complex, produced by Photocor Instruments Inc.). A 654-nm diode laser was used as the light source throughout the experiment, and scattered light was measured by an avalanche photodiode (APD).

A polarizer was installed in front of the sample and another polarizer was kept after the sample which passes laser light to the APD. The incident light before the sample and the scattered light measured by the APD both were vertically polarized (VV) for DLS measurements. The incident light was vertically polarized and horizontal polarization was selected from the scattered light (VH) for DDLS measurements. For the DLS measurements of dissolved BPM and Salol, the scattering angles were $30^{\circ}, 45^{\circ}, 60^{\circ}$, and $75^{\circ}$, respectively. All samples were kept in $15-\mathrm{mm}$-diameter cylindrical glass bottles and heated at 280,300 , and $320 \mathrm{~K}$ by a thermostat with an accuracy of $0.1 \mathrm{~K}$. The DDLS measurements of dissolved BPM and Salol were taken at much lower scattering angles of $25^{\circ}, 30^{\circ}, 35^{\circ}$, and $40^{\circ}$ at $280 \mathrm{~K}$ because of the low acceptance and weakness of the depolarized signal compared to the polarized signal.

TABLE I. $\beta$-relaxation time of BPM and Salol in Deuterated acetone obtained from the QENS data.

\begin{tabular}{lcc}
\hline \hline \multicolumn{3}{c}{ QENS Measurement $\beta$-relaxation Time $\left(10^{-13} \mathrm{~s}\right)$} \\
\hline Temperature (K) & Dissolved BPM & Dissolved Salol \\
\hline $213 \mathrm{~K}$ & $0.36 \pm 0.01$ & $1.92 \pm 0.03$ \\
$228 \mathrm{~K}$ & $0.99 \pm 0.02$ & $2.52 \pm 0.05$ \\
$243 \mathrm{~K}$ & $1.80 \pm 0.04$ & $3.17 \pm 0.06$ \\
$258 \mathrm{~K}$ & $1.92 \pm 0.04$ & $3.90 \pm 0.07$ \\
$273 \mathrm{~K}$ & $2.62 \pm 0.06$ & $4.36 \pm 0.09$ \\
\hline \hline
\end{tabular}

The DDLS measurements of bulk BPM and Salol were taken with scattering angles of $25^{\circ}, 30^{\circ}, 35^{\circ}$, and $40^{\circ}$ at $340 \mathrm{~K}$ and $15^{\circ}, 20^{\circ}, 22.5^{\circ}$, and $25^{\circ}$ at $300 \mathrm{~K}$, respectively. These measurements have a range of scattering vector $Q$, which can be calculated by equation $Q=4 \pi n \sin (\theta / 2) / \lambda$, where $n$ is the refractive index of the solution, $\lambda$ is the wavelength of the light source, and $\theta$ is the scattering angle. The refractive indexes of bulk BPM, Salol, and acetone are 1.595, 1.615, and 1.359, respectively. According to the Lorentz-Lorenz (LL) relation [29] for calculating the refractive index of binary liquid mixtures, we obtained refractive indexes of 1.440 and 1.447 for a BPM and acetone mixture (molecular ratio 1:4) and a Salol and acetone mixture (molecular ratio 1:4), respectively. When laser light goes through the acetone solutions of BPM or Salol, the light scatters due to variation of the refractive index and fluctuation of the scattered light intensity over time due to the motion of the BPM or Salol molecules in the solution.

\section{RESULTS AND DISCUSSION}

The relaxation dynamics of BPM and Salol molecules within the deuterated acetone can be studied by the present QENS experiments. Figure 2 shows the dynamic structure factors of dissolved BPM in deuterated acetone measured at different temperatures. The resulting dynamic structure factors were Fourier transformed, divided by the resolution function, and normalized at $t=0$ to obtain the self-intermediate scattering function, $\Phi(Q, t)$.

The QENS experiments observed the logarithmic decays of $\Phi(Q, t)$ of BPM and Salol dissolved in deuterated

TABLE II. $\beta$-relaxation time of bulk BPM and Salol obtained from the QENS data.

\begin{tabular}{lccc}
\hline \hline \multicolumn{4}{c}{ QENS Measurement $\beta$-relaxation Time $\left(10^{-13} \mathrm{~s}\right)$} \\
\hline Temperature (K) & Bulk BPM & Temperature $(\mathrm{K})$ & Bulk Salol \\
\hline $313 \mathrm{~K}$ & $26.1 \pm 0.29$ & $325 \mathrm{~K}$ & $0.13 \pm 0.02$ \\
$323 \mathrm{~K}$ & $33.3 \pm 0.41$ & $350 \mathrm{~K}$ & $0.34 \pm 0.02$ \\
$333 \mathrm{~K}$ & $31.6 \pm 0.33$ & $375 \mathrm{~K}$ & $1.04 \pm 0.04$ \\
$343 \mathrm{~K}$ & $41.0 \pm 0.62$ & $400 \mathrm{~K}$ & $1.20 \pm 0.03$ \\
\hline \hline
\end{tabular}



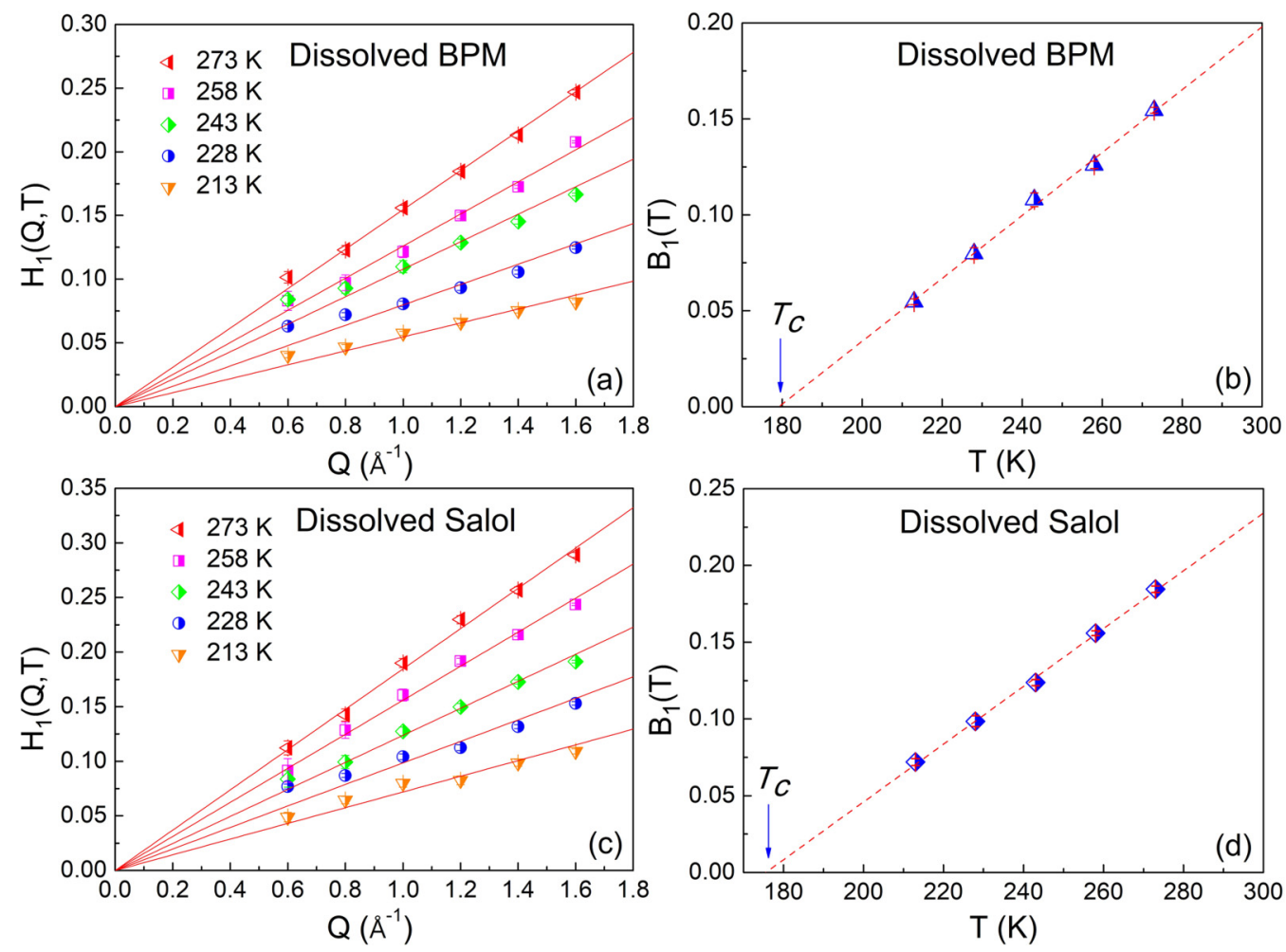

FIG. 6. The QENS fitting parameters of dissolved BPM and Salol. (a) $\mathrm{H}_{1}(Q, T)$ of dissolved BPM and (c) dissolved Salol as a function of $Q$ at different temperatures. The temperature dependence of fitting parameter $\mathrm{B}_{1}(T)$ of (b) dissolved BPM and (d) dissolved Salol. The linear fitting lines are extrapolated consistently to get the MCT critical temperature, $T_{c}$.

acetone measured at various temperatures [see Figs. 3(a) and 3(b)]. Figures 4(a) and 4(b) show $\Phi(Q, t)$ for BPM and Salol samples dissolved in deuterated acetone measured at various $Q$ values and at a temperature of $243 \mathrm{~K}$. Figures 5(a) and 5(b) show $\Phi(Q, t)$ for bulk BPM and Salol measured at various temperatures [30]. These spectra clearly show that on a semilogarithmic scale, $\Phi(Q, t)$ decays in a straight line in the time window from $8 \times 10^{-13} \mathrm{~s}$ to $1.5 \times 10^{-11} \mathrm{~s}$. This indicates that BMP and Salol molecules still exhibit logarithmic decay of the correlation for the fast $\beta$-relaxation process, although the intermolecular potential is significantly changed in the dissolved state. In the optical heterodyne-detected optical Kerr effect (OHD-OKE) mesearments on BPM and Salol, the $\alpha$ relaxation process occurs in the time scale $\gtrsim 1 \mathrm{~ns}$ [14]. Such a slow time scale cannot be achieved by time-of-flight neutron scattering instruments. The time scale of the present QENS experiments can therefore probe the fast $\beta$-relaxation process. The results indicate that the dissolved state does not hinder the logarithmic relaxation process. In this dissolved state, a BPM or a Salol molecule is surrounded by several deuterated acetone molecules and solute molecules. Therefore, the intermolecular potential must be drastically different from that of bulk BPM or bulk Salol. Thus, we can confirm that the intermolecular potential does not play a role in determining the logarithmic relaxation process.

To understand how the dissolved state affects the fast $\beta$ relaxation time scale and microscopic glass-transition temperature $\left(T_{c}\right)$, the following asymptotic formula derived by the
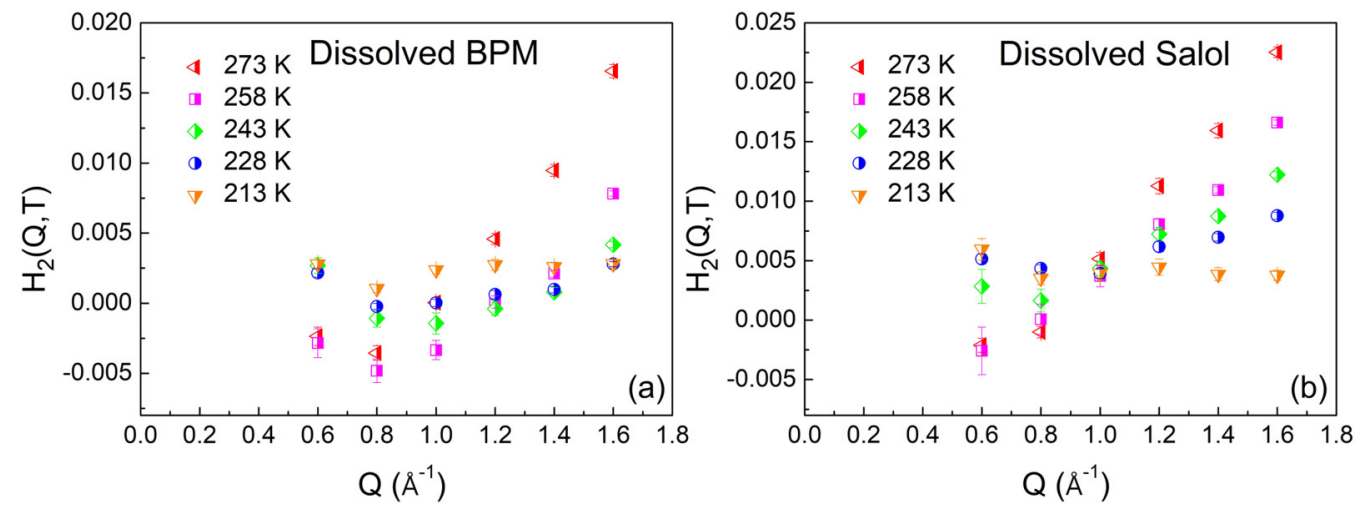

FIG. 7. The fitting parameter $\mathrm{H}_{2}(Q, T)$ of (a) dissolved BPM and (b) dissolved Salol as a function of $Q$ at different temperatures. 
TABLE III. Mean relaxation time of $\alpha$-relaxation process of BPM and Salol molecules in the bulk state and dissolved state.

\begin{tabular}{lr}
\hline \hline \multicolumn{1}{c}{ DLS Measurement $\alpha$-relaxation time $\left(10^{-3} \mathrm{~s}\right)$} \\
\hline Temperature: $320 \mathrm{~K}$ & \multicolumn{1}{c}{$Q=0.001 \AA^{-1}$} \\
\hline Bulk BPM & $138.35 \pm 1.05$ \\
Dissolved BPM & $9.47 \pm 0.21$ \\
Bulk Salol & $44.31 \pm 0.69$ \\
Dissolved Salol & $2.13 \pm 0.01$ \\
\hline \hline
\end{tabular}

MCT was used to analyze $\Phi(Q, t)$ obtained from the QENS experiments [20,30-32]:

$$
\begin{aligned}
\Phi(Q, t) \approx & {\left[f(Q, T)-H_{1}(Q, T) \ln \left(t / \tau_{\beta}(T)\right)\right.} \\
& \left.+\mathrm{H}_{2}(Q, T) \ln ^{2}\left(\mathrm{t} / \tau_{\beta}(\mathrm{T})\right)\right],
\end{aligned}
$$

where $f(Q, T)$ is the Debye-Waller factor, $\tau_{\beta}(T)$ is the characteristic $\beta$-relaxation time, which is independent of $Q$. $\mathrm{H}_{1}(Q, T)$ and $\mathrm{H}_{2}(Q, T)$ represent the first- and second-order logarithmic decay parameters, respectively. The $\beta$-relaxation times of BPM and Salol in Deuterated acetone are noted in Table I and that of bulk BPM and Salol are shown in Table II. The $Q$ dependence of the parameter $\mathrm{H}_{1}(Q, T)$ is shown in Figs. 6(a) and 6(c). The solid lines are the fits with the following equation: $\mathrm{H}_{1}(Q, T)=\mathrm{B}_{1}(T) Q$ [20]. The values of $\mathrm{B}_{1}(T)$ obtained from the fitting were plotted as a function of temperature in Figs. 6(b) and 6(d). The temperature dependence of $\mathrm{B}_{1}(T)$ is extrapolated consistently to obtain $T_{c}$. At $T_{c}$ the transport mechanism changes from liquid-like molecular motion to glass-like activated hopping processes [12,33]. $T_{c}$ of bulk BPM is found to be $282 \pm 2 \mathrm{~K}$, which is $\sim 10 \mathrm{~K}$ lower than the value $(290 \pm 4 \mathrm{~K})$ in previous studies [13,14]. This $T_{c}$ value is approximately $1.2 T_{g}\left(T_{g} \sim 239 \mathrm{~K}\right)$, which is observed for many glass-forming liquids [34]. $T_{c}$ of bulk Salol is found to be $255 \pm 2 \mathrm{~K}$ in our previous study [30]. The $T_{c}$ values of dissolved BPM and Salol are $180 \pm 2 \mathrm{~K}$ and 176 $\pm 2 \mathrm{~K}$, respectively, which are shifted $\sim 100 \mathrm{~K}$ and $\sim 80 \mathrm{~K}$ to lower temperatures compared with the bulk samples. This shows that dynamic transition occurs at a much lower temperature in the dissolved state than in the bulk state. This indicates the intermolecular potential experienced by Salol and BPM molecules in the dissolved state must be different from
TABLE IV. Translational diffusion coefficient of dissolved BPM and Salol obtained by the dynamic light scattering measurements.

\begin{tabular}{lcc}
\hline \hline & Dissolved BPM & Dissolved Salol \\
Temperature $(\mathrm{K})$ & $D_{T}\left(10^{-13} \mathrm{~m}^{2} \mathrm{~s}^{-1}\right)$ & $D_{T}\left(10^{-13} \mathrm{~m}^{2} \mathrm{~s}^{-1}\right)$ \\
\hline 320 & $11.467 \pm 0.398$ & $40.645 \pm 1.217$ \\
300 & $8.106 \pm 0.117$ & $35.110 \pm 0.847$ \\
280 & $6.488 \pm 0.157$ & $27.406 \pm 0.820$ \\
\hline \hline
\end{tabular}

that of the bulk state. For the dissolved samples, $\mathrm{H}_{2}(Q, T)$ parameters are shown in Fig. 7. In order to check the possibility of a long stretched exponential decay or a power-law decay we have fitted the $\Phi(Q, t)$ of dissolved samples using the above functions. The fitting of above functions to the data of $\Phi(Q, t)$ were very poor, see the Supplemental Material, Fig. $1 S$ and Table 1S [35]. Therefore, we confirm the logarithmic decay of self-intermediate scattering function of dissolved BPM and Salol.

The fluctuations of the scattered light can be analyzed by measuring the intensity-intensity ACF:

$$
g_{2}(Q, t)=\frac{1}{\langle I\rangle^{2}} \lim _{T \rightarrow \infty} \frac{1}{T} \int_{0}^{T} I(Q, t) I(Q, t+\tau) d \tau-1,
$$

where $I$ is the scattered light intensity, $\langle I\rangle$ is the temporal average scattered light intensity, $T$ is the acquisition time, and $Q$ is the scattering vector. The autocorrelation function $g_{2}(Q, t)$ is related to the desired electric field time autocorrelation function $g_{1}(Q, t)$ through the Siegert relation, $g_{2}(t)=I^{2}\left(1+f\left|g_{1}(t)\right|^{2}\right)$, where $f$ is the optical factor of the instrument.

In the DLS and DDLS measurements, the autocorrelation functions can be fitted with the Kohlrausch function (see Figs. 3(c) and 3(d) [36]:

$$
g_{2}(Q, t)=f_{Q} \exp \left[-\left(t / \tau_{\alpha}\right)^{\beta}\right],
$$

where $f_{Q}$ is the Debye-Waller factor, $\tau_{\alpha}$ is the $\alpha$-relaxation time, and $\beta$ is the stretching exponent. The mean relaxation time $\left\langle\tau_{\alpha}\right\rangle$ can be calculated by $\left\langle\tau_{\alpha}\right\rangle=\frac{\tau_{\alpha}}{\beta} \Gamma\left(\frac{1}{\beta}\right)$, where $\Gamma$ is the gamma function. The mean relaxation time of the $\alpha$-relaxation process of BPM and Salol molecules in the bulk state and
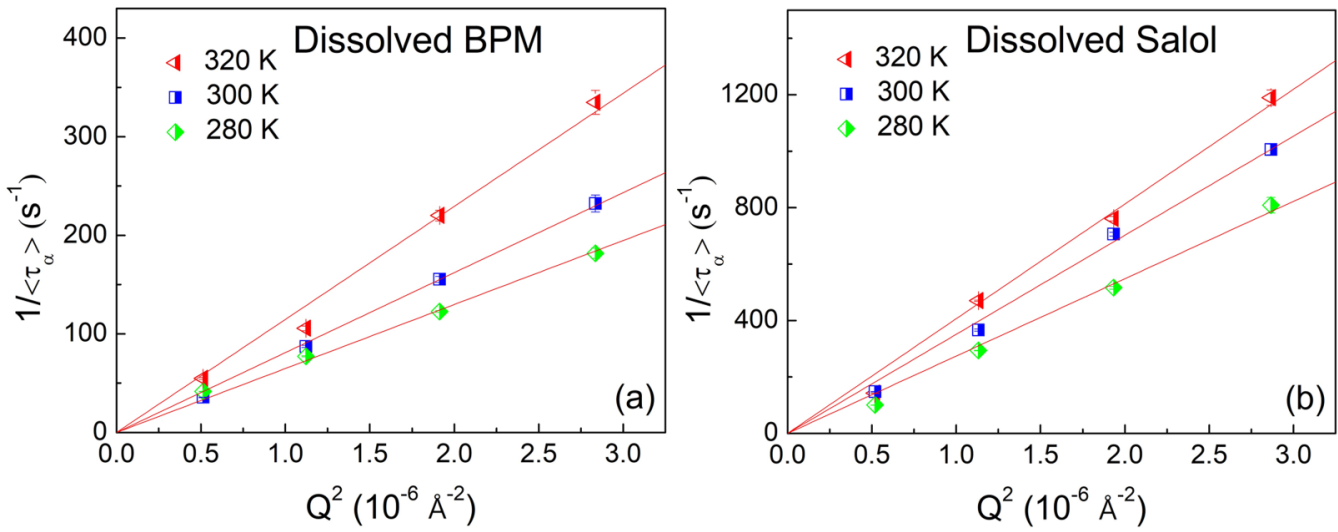

FIG. 8. The inverse of mean $\alpha$-relaxation time of dissolved (a) BPM and (b) Salol are plotted as a function of $Q^{2}$. 

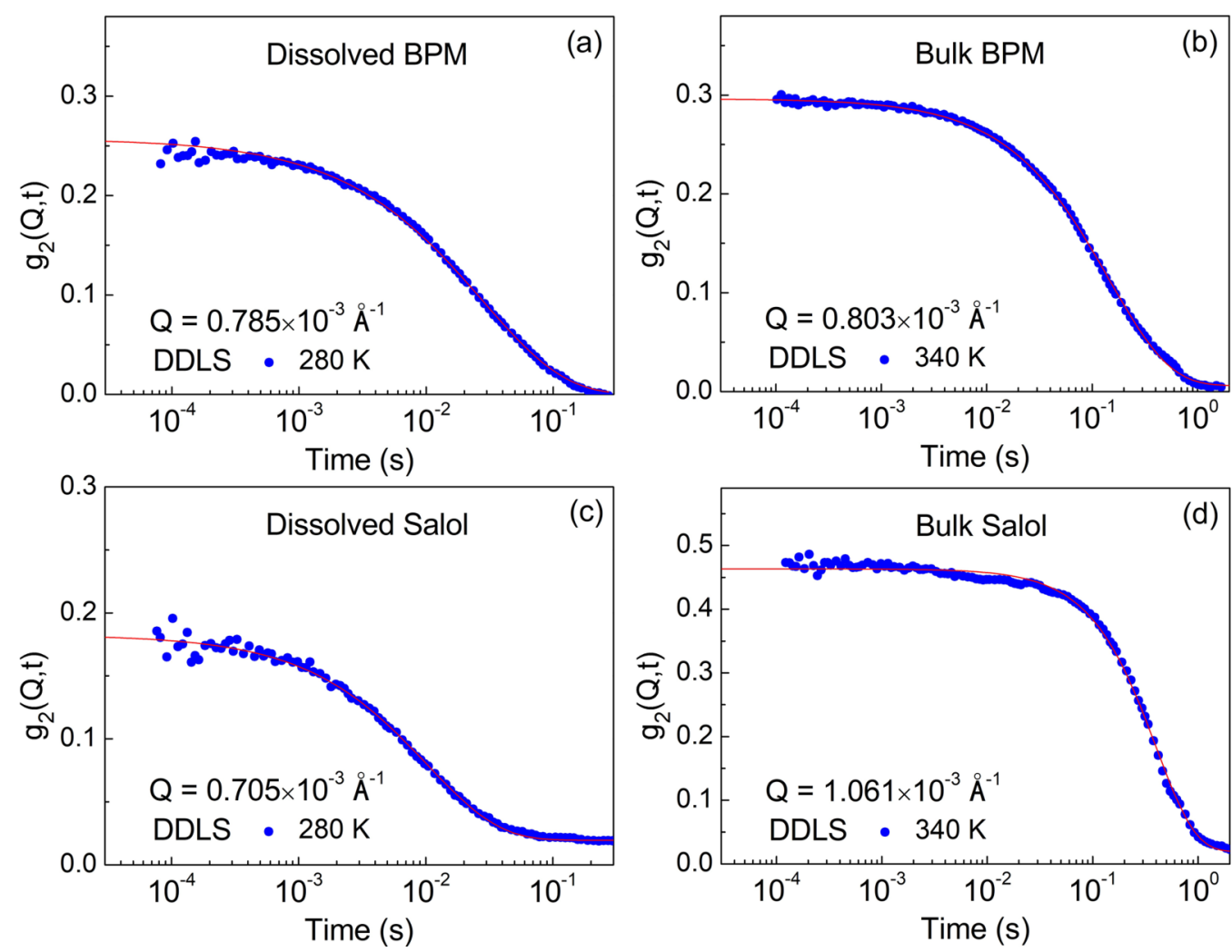

FIG. 9. The intensity-intensity autocorrelation functions obtained by DDLS for (a) dissolved BPM, (b) bulk BPM, (c) dissolved Salol and (d) bulk Salol, respectively. The solid lines are the fits with Kohlrausch function [Eq. (3)].

dissolved state are noted in Table III. The inverse of the mean $\alpha$-relaxation time obtained by the DLS measurements of dissolved BPM and Salol are plotted as a function of $Q^{2}$ in Fig. 8. The translational diffusion coefficients of these samples can be calculated using the following equation [37], and the values are shown in Table IV:

$$
D_{T}=\lim _{Q \rightarrow 0} \frac{1}{\left\langle\tau_{\alpha}\right\rangle Q^{2}} .
$$

Figures 9(a) and 9(c) show the intensity-intensity autocorrelation function, $g_{2}(Q, t)$, obtained by DDLS experiments on the BPM and Salol in acetone and Figs. 9(b) and 9(d) show $g_{2}(Q, t)$ of bulk BPM and Salol. The time scale of the DLS experiments is on the order of microseconds, and the $\alpha$-relaxation processes of the BMP and Salol molecules is clearly visible on this time scale.

Götze and Sperl [17,19] showed that logarithmic decay of the correlation function can be obtained from a twocorrelator model of MCT. One of the correlators is the density fluctuations, and the other is the reorientational fluctuations of molecules. The model resulted in a strong translationalrotational coupling. In bulk BPM, the hydroxyl groups $(-\mathrm{OH})$ of the BPM molecule form intermolecular hydrogen bonds $[21,38]$. In the dissolved state, the BPM molecules are surrounded by deuterated acetone, so the interactions and potential between molecules are different. The results of the dissolved BPM have addressed the important question of whether the logarithmic relaxation still exists in the dissolved state, even though the potentials of the BPM molecules have changed from that of bulk BPM molecules. Therefore, the origin of logarithmic relaxation must not be the interatomic potentials between BPM-BPM or Salol-Salol molecules. Another possibility is the rotational-translational coupling. To examine this possibility, we evaluated the rotational and translational diffusion coefficient of Salol and BPM molecules in their bulk state and in deuterated acetone.

Figures 10(a)-10(d) show the inverse of the mean $\alpha$ relaxation time of BPM and Salol in deuterated acetone and bulk states plotted as a function of $Q^{2}$ obtained from the DDLS measurements. The solid straight lines were fitted with the following equation [39]:

$$
\frac{1}{\left\langle\tau_{\alpha}\right\rangle}=Q^{2} D_{T}+6 D_{R},
$$

where $\left\langle\tau_{\alpha}\right\rangle$ is the mean $\alpha$-relaxation time obtained from the Kohlrausch function fitting of the DDLS autocorrelation function (see Fig. S6) and fitting parameters are listed in the Table V. $D_{T}$ and $D_{R}$ are the translational and rotational

TABLE V. $\alpha$-relaxation time and stretching exponent $(\beta)$ values of BPM and Salol in bulk and in dissolved states obtained by depolarized dynamic light scattering measurements.

\begin{tabular}{lrc}
\hline \hline & $\alpha$-relaxation Time $\left(10^{-3} \mathrm{~s}\right)$ & $\beta$ \\
\hline Dissolved BPM (280 K) & $27.74 \pm 0.72$ & $0.67 \pm 0.02$ \\
Bulk BPM (340 K) & $155.30 \pm 2.26$ & $0.81 \pm 0.01$ \\
Dissolved Salol (280 K) & $10.31 \pm 0.23$ & $0.77 \pm 0.02$ \\
Bulk Salol (340 K) & $401.45 \pm 5.38$ & $0.98 \pm 0.02$ \\
\hline \hline
\end{tabular}



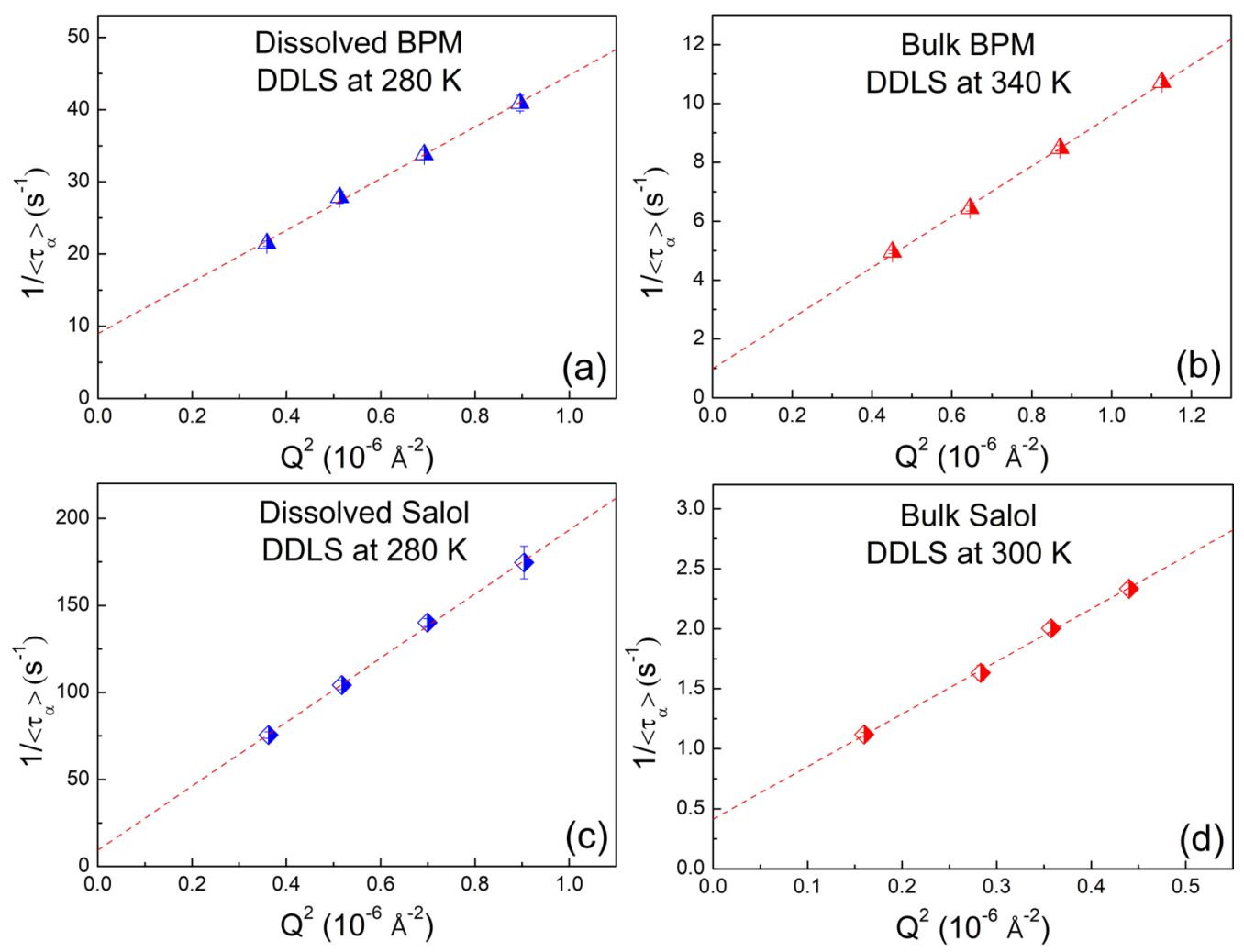

FIG. 10. The DDLS data of BPM and Salol in dissolved and bulk states. The inverse of the mean $\alpha$-relaxation time of (a) dissolved BPM, (b) bulk BPM, (c) dissolved Salol, and (d) bulk Salol as a function of square of momentum transfer, $Q^{2}$.

diffusion coefficients, respectively. The $D_{T}$ and $D_{R}$ values for all the samples are shown in Table VI. The diffusion in the dissolved state is faster than in the bulk samples. The ratios of the translational and rotational diffusion coefficients of bulk BPM compared with that of dissolved BPM are 0.240 and 0.111, and those for Salol samples are 0.024 and 0.045 , respectively. Thus, the ratio of the translational to rotational diffusion coefficient does not show a significant change. This suggests that the rotational-translational coupling could be possible in the dissolved state also. Additionally, the $\alpha$-relaxation process is slightly stretched in the dissolved state than in the bulk samples (e.g., the stretching exponent $\beta$ is 0.67 for dissolved BPM and 0.87 for bulk BPM with scattering angle of $45^{\circ}$ at $320 \mathrm{~K}$ ). Similarly, the time scale of the fast $\beta$-relaxation in the bulk Salol and BPM is on the order of picoseconds to nanoseconds. In deuterated acetone, the fast $\beta$-relaxation is still visible at the same time scale. This indicates that the

TABLE VI. The translational and rotational diffusion coefficients of BPM and Salol in the dissolved and bulk states obtained by the DDLS measurements.

\begin{tabular}{lcrc}
\hline \hline Samples & $\begin{array}{c}\text { Temperature } \\
(\mathrm{K})\end{array}$ & $\begin{array}{c}D_{T} \\
\left(10^{-13} \mathrm{~m}^{2} \mathrm{~s}^{-1}\right)\end{array}$ & \multicolumn{1}{c}{$D_{R}\left(\mathrm{~s}^{-1}\right)$} \\
\hline Dissolved BPM & 280 & $3.579 \pm 0.113$ & $1.498 \pm 0.122$ \\
Bulk BPM & 340 & $0.860 \pm 0.019$ & $0.166 \pm 0.025$ \\
Dissolved Salol & 280 & $18.408 \pm 0.449$ & $1.550 \pm 0.489$ \\
Bulk Salol & 340 & $0.438 \pm 0.011$ & $0.069 \pm 0.004$ \\
\hline \hline
\end{tabular}

translational-rotational coupling in the bulk samples must be present in the samples dissolved in deuterated acetone.

\section{CONCLUSION}

In summary, we have studied the molecular relaxation process of BPM and Salol in their bulk and dissolved states using QENS and DDLS. The fast $\beta$-relaxation process of these molecules is logarithmic with respect to time. The $\operatorname{logarithmic}$ nature of the fast $\beta$-relaxation process was found in the dissolved state, although the intermolecular potentials of the BPM and Salol molecules were drastically different in the dissolved state compared to the bulk state. The MCT critical temperatures of dissolved BPM and Salol were both reduced, showing that dynamic transition occurs at a much lower temperature in the dissolved state than in the bulk state. However, the ratio of the translational-to-rotational diffusion coefficients does not show appreciable change. This indicates that the logarithmic relaxation exhibited by the BPM and Salol likely due to the coupling of translational-rotational relaxation processes.

\section{ACKNOWLEDGMENTS}

This research has been supported by City University Strategic Research Grant No. 7004546. All authors thank the Australian National Nuclear Research and Development Organisation for the neutron scattering beam time on Pelican time-of-flight neutron scattering spectrometer and the Paul Scherrer Institute, Switzerland for beam time on the MARS backscattering spectrometer. 
[1] C. A. Angell, K. L. Ngai, G. B. McKenna, P. F. McMillan, and S. W. Martin, J. Appl. Phys. 88, 3113 (2000).

[2] M. H. Bhat, V. Molinero, E. Soignard, V. C. Solomon, S. Sastry, J. L. Yarger, and C. A. Angell, Nature (London) 448, 787 (2007).

[3] C. A. Angell, Chem. Rev. 102, 2627 (2002).

[4] J. Swenson and S. Cerveny, J. Phys. Condens. Matter 27, 033102 (2015).

[5] L. Hong, V. N. Novikov, and A. P. Sokolov, Phys. Rev. E 83, 061508 (2011).

[6] A. Meyer, Phys. Rev. B 66, 134205 (2002).

[7] L. Orsingher, A. Fontana, E. Gilioli, G. Carini, G. Carini, G. Tripodo, T. Unruh, and U. Buchenau, J. Chem. Phys. 132, 124508 (2010).

[8] Z. Wang, Q. Lv, S. Chen, C. Li, S. Sun, and S. Hu, Mol. Simul. 41, 1515 (2015).

[9] M. T. Cicerone, D. Averett, and J. J. De Pablo, J. Non. Cryst. Solids 407, 118 (2015).

[10] F. Faupel, K. Ratzke, M. P. Machat, H. Mehrer, N. Naundrof, K. Ratzke, H. R. Schober, and K. S. Sharma, Rev. Mod. Phys. 75, 237 (2003).

[11] M. T. Cicerone, Q. Zhong, and M. Tyagi, Phys. Rev. Lett. 113, 117801 (2014).

[12] G. Hinze, D. D. Brace, S. D. Gottke, and M. D. Fayer, Phys. Rev. Lett. 84, 4783 (2000).

[13] H. Cang, V. N. Novikov, and M. D. Fayer, Phys. Rev. Lett. 90, 197401 (2003)

[14] H. Cang, V. N. Novikov, and M. D. Fayer, J. Chem. Phys. 118, 2800 (2003).

[15] X. Chu, M. Lagi, E. Mamontov, E. Fratini, P. Baglioni, and S.-H. Chen, Soft Matter 6, 2623 (2010).

[16] X. Chu, E. Mamontov, H. O'Neill, and Q. Zhang, J. Phys. Chem. Lett. 4, 936 (2013).

[17] W. Götze and M. Sperl, Phys. Rev. Lett. 92, 105701 (2004).

[18] G. Li, W. M. Du, A. Sakai, and H. Z. Cummins, Phys. Rev. A 46, 3343 (1992).

[19] M. Sperl, Phys. Rev. E 74, 011503 (2006).
[20] W. Götze and M. Sperl, Phys. Rev. E 66, 011405 (2002).

[21] J. Baran, N. A. Davydova, and M. Drozd, J. Mol. Liq. 127, 109 (2006).

[22] Y. Yang and K. A. Nelson, Phys. Rev. Lett. 74, 4883 (1995).

[23] D. Yu, R. Mole, T. Noakes, S. Kennedy, and R. Robinson, J. Phys. Soc. Jpn. 82, SA027 (2013).

[24] F. Kargl, H. Weis, T. Unruh, and A. Meyer, J. Phys. Conf. Ser. 340, 012077 (2012).

[25] S. M. Chathoth and A. Podlesnyak, J. Appl. Phys. 103, 013509 (2008).

[26] D. Richard, M. Ferrand, and G. J. Kearley, J. Neutron Res. 4, 33 (1996).

[27] P. L. W. Tregenna-Piggott, F. Juranyi, and P. Allenspach, Neutron News 19, 20 (2008).

[28] R. T. Azuah, L. R. Kneller, Y. Qiu, P. L. W. Tregenna-Piggott, C. M. Brown, J. R. D. Copley, and R. M. Dimeo, J. Res. Natl. Inst. Stand. Technol. 114, 341 (2009).

[29] T. M. Aminabhavi, J. Chem. Eng. Data 29, 54 (1984).

[30] C. Chen, K. Wong, R. A. Mole, D. Yu, and S. M. Chathoth, Sci. Rep. 6, 33374 (2016).

[31] M. Lagi, P. Baglioni, and S.-H. Chen, Phys. Rev. Lett. 103, 108102 (2009).

[32] M. Sperl, Phys. Rev. E. 68, 031405 (2003).

[33] W. Götze and L. Sjögren, Transp. Theory Stat. Phys. 24, 801 (1995).

[34] R. Bohmer, K. L. Ngai, C. A. Angell, and D. J. Plazek, J. Chem. Phys. 99, 4201 (1993).

[35] See Supplemental Material at http://link.aps.org/supplemental/ 10.1103/PhysRevB.98.094203 for more details of KWW and power-law fitting.

[36] M. Cardona, R. V. Chamberlin, and W. Marx, Ann. Der Phys. 16, 842 (2007).

[37] K. S. Singwi and A. Sjölander, Phys. Rev. 119, 863 (1960).

[38] J. Baran, N. A. Davydova, M. Drozd, and A. Pietraszko, J. Phys. Condens. Matter 18, 5695 (2006).

[39] D. Lehner, H. Lindner, and O. Glatter, Langmuir 16, 1689 (2000). 